\title{
Optical fiber laser ultrasound transmitter with electrospun composite for minimally invasive medical imaging
}

\author{
Radhika Poduval $^{1}$, Sacha Noimark ${ }^{2,3}$, Richard Colchester ${ }^{2}$, Tom Macdonald ${ }^{3}$, \\ Ivan Parkin ${ }^{3}$, Adrien Desjardins ${ }^{2}$, Ioannis Papakonstantinou ${ }^{1}$ \\ ${ }^{1}$ Department of Electronic and Electrical Engineering, University College London, London WC1E 7JE, U.K. \\ ${ }^{2}$ Department of Medical Physics and Biomedical Engineering, University College London, London WC1E 6BT, U.K. \\ ${ }^{3}$ Department of Chemistry, University College London, London WC1H OAJ, U.K.. \\ email : i.papakonstantinou@ucl.ac.uk
}

\begin{abstract}
We report an optical fiber ultrasound transmitter with electrospun MWCNT-polymer composite, generating high-amplitude broadband ultrasound. They produced pressures in the range of conventional intravascular imaging transducers, and can be incorporated into catheters/needles for keyhole surgery.
\end{abstract}

OCIS codes: $170.0170,110.0110,160.0160$

\section{Introduction}

Nanostructured devices based on optical fibers present diverse application possibilities. Optical ultrasound imaging for minimally invasive surgery (MIS) is one such area, where ultrasound is transmitted and received optically. Piezoelectric transducers, and to some extent capacitive micro-machined transducers are currently used for ultrasound imaging. All-optical ultrasound imaging probes could help further miniaturise and reduce the cost of ultrasound transducers for pulse-echo ultrasound imaging in MIS. In addition to providing electromagnetic noise immunity [1] optical ultrasound devices could be designed to posses improved sensitivities and bandwidths, with the potential to easily scale-up the probe fabrication process.

Composites of carbonaceous optical absorbers in polydimethylsiloxane (PDMS) elastomeric hosts are particularly suited to photoacoustic generation of pressures in the MPa range that are of interest for biomedical pulse-echo ultrasound imaging [1-5]. These transmitters are able to produce ultrasound pressures and bandwidths comparable to those of conventional intravascular ultrasound imaging transducers. Although optical ultrasound generation has been reported recently through composite dip-coating at the endface of optical fibers cleaved at normal incidence [3, 4], control of the absorber layer coating thickness is difficult with this method. The coating thickness directly influences the ultrasound attenuation within it, and consequently the ultrasound generation efficiency of the transmitter. Additionally, it is desirable to improve PDMS infiltration into the multi-walled carbon nanotube (MWCNTs) mesh, i.e. the optically absorbing component of the composite, for efficient heat transfer between the nanotubes and PDMS [6].

Thickness control and elastomer infiltration into the optically absorbing component is addressed here by employing a nanofibrous, porous absorber element. We used a simple and scalable technique involving electrospinning to form a nanofibrous optical absorber consisting of multi-walled carbon nanotubes (MWCNTs) in polyvinyl alcohol (PVA), to act as the optically absorbing element in our fiber optic ultrasound transmitter. The porous nature of the electrospun MWCNT nanofibers can function to promote the elastomer infiltration. Furthermore, optical absorption anisotropy of MWCNTs is established in literature, stating that the absorption cross-section of MWCNTs almost doubles when the incident illumination is co-polarised with the MWCNT axis, compared to the cross-polarised condition [7, 8]. The planar arrangement of MWCNTs within the electrospun nanofibers, when illuminated by the plane plane polarised light emerging from optical fiber tip is expected to enhance the efficiency of ultrasound generation by the fiber optic transmitter.

Opto-Acoustic Methods and Applications in Biophotonics III, edited by Vasilis Ntziachristos,

Roger Zemp, Proc. of SPIE-OSA Vol. 10415, 104150B · (c) 2017 SPIE-OSA

CCC code: $1605-7422 / 17 / \$ 18 \cdot$ doi: $10.1117 / 12.2286162$ 


\section{Fabrication methods}

Here we describe a novel fabrication method for optical ultrasound transmitters through deposition of an electrospun MWCNT composite on an optical fiber tip. The steps involved in the fabrication of these miniature ultrasound transmitters is illustrated in Fig.1. The process consisted of three steps - electrospinning the absorber, transfer of absorber mat onto the optical fiber, and finally incorporation of elastomer. MWCNT solution with poly-vinyl alcohol (PVA) as the carrier polymer was electrospun onto a flat collector covered with aluminium foil . The aluminium foil containing the electrospun nanofibers was then removed from the flat frame and a small tear was made around the central region of the foil, which was carefully pulled away to expose the CNT-PVA nanofiber mat. The nanofibers were then transferred onto the optical fiber by insertion of its cleaved endface through the nanofiber mat. This was followed by dip coating in PDMS elastomer (Sylgard 184). Since the deposition area of the nanofibers on the flat aluminium collector is much larger than the cross-sectional area of the optical fiber, this method can be used for depositing electrospun fibers simultaneously on multiple optical fiber endfaces, therefore facilitating the fabrication process scale-up of electrospun optical fiber-based devices.

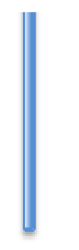
Optical fiber cleaved at
normal incidence

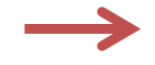

MWCNT-PVA mat electrospun transferred onto optical fiber end-face

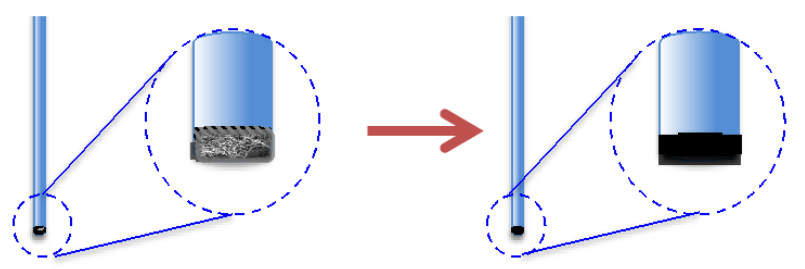

Optical fiber tip on dip composite for ultrasound generation

Fig. 1: (a) Fabrication sequence for preparing the fiber optic ultrasound transmitter through MWCNT electrospinning

The electrospinning precursor solution was prepared by mixing an aqueous MWCNT dispersion in 9 wt.\% solution of PVA (Sigma Aldrich 321584-25G, molecular wt. 8900098 000, $99 \%$ hydrolyzed in deionised water. For this preparation, MWCNTs with dimensions of 6-9 $\mathrm{nm}$ x $5 \mu \mathrm{m}$ (724769, Sigma Aldrich, UK) were functionalized and dispersed in deionized water ( $30 \mathrm{ml}$ water) using cetyltrimethylammonium bromide (CTAB, $0.3 \mathrm{~g}$ ) through a modification of the method described by [9]. The electrospinning precursor preparation used is as detailed in [10], where electrospinning was performed directly onto a single optical fiber endface. But here, we make the use of electrospun absorber scalable through electrospinning on a large area (electrospinning time $12 \mathrm{mins}$ ), and transferring sections of this coating onto the optical fiber endface by piercing the cleaved optical fiber through the electrospun MWCNT composite.

Elastomer infiltration into the transferred electrospun mat was performed by dip coating. The previously prepared optical fiber containing the electrospun MWCNT-PVA composite was dip coated in a Sylgard 186 polymer solution diluted with $30 \mathrm{wt} . \%$ of toluene to lower elastomer viscosity and promote elastomer infiltration into the pores of the electrospun coating layer. Following dip-coating, the optical fiber endface was left to cure facing upright at room temperature for $48 \mathrm{~h}$, following which the optical and ultrasound characterisation were performed.

\section{Results and discussion}

The above detailed fabrication process produced uniform coverage of the composite coating on the core region of the optical fiber endface through scanning electron microscopy (SEM), as depicted in Fig 1 (b). Furthermore, complete infiltration of dip coated PDMS elastomer was observed through cross-section SEM of the coating. Additionally, the preferential alignment of the MWCNTs along the PVA nanofibers was also observed through transmission electron microscopy (TEM) of the electrospun nanofibers.

The prepared transmitters were evaluated to determine their optical and ultrasound characteristics. Optical absorption was measured in an integrating sphere setup (FOIS-1, Ocean Optics, USA). The ultrasound generation and pressure measurements we conducted using the method described in [11] (the setup is schematically shown in Fig. 2 (a)).The excitation light was provided by a Q-switched Nd:YAG $1064 \mathrm{~nm}$ laser (SPOT-105001064, Elforlight, UK), with a pulse width of $2 \mathrm{~ns}$, a repetition rate of $100 \mathrm{~Hz}$, and a laser pulse energy of $13 \mu \mathrm{J}$ (fluence: $41.4 \mathrm{~mJ} / \mathrm{cm} 2$ ). For pressure measurements, the optical fiber ultrasound transmitters were positioned in water facing a calibrated 75 $\mu \mathrm{m}$ diameter needle hydrophone (SN 493, Precision Acoustics, UK) with a calibration range of $130 \mathrm{MHz}$ and $1.5 \mathrm{~mm}$ separation between the transmitter and the hydrophone surface. 
Optical absorption of the fiber optic ultrasound transmitter was measured to be 0.96 at $1064 \mathrm{~nm}$. On providing the excitation pulses to the transmitters as previously described, the peak-to-peak pressure at $1.5 \mathrm{~mm}$ from the fiber tip was measured as $1.16 \mathrm{MPa}$ for the fiber optic electrospun ultrasound transmitter. Furthermore the $-6 \mathrm{~dB}$ bandwidth was $25 \mathrm{MHz}$ with signal peak at $24.3 \mathrm{MHz}$ for. These values of ultrasound pressure and bandwidth are suitable for intravascular tissue imaging. To cater to other applications where higher pressure values are required, either the incident laser pulse fluence can be increased or the coating could be further optimised to further reduce local ultrasound attenuation.

(a)

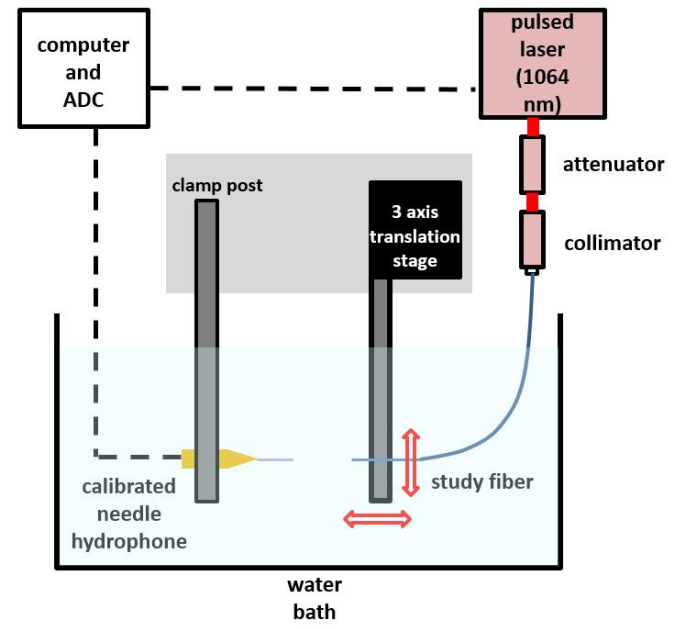

(b)

i.

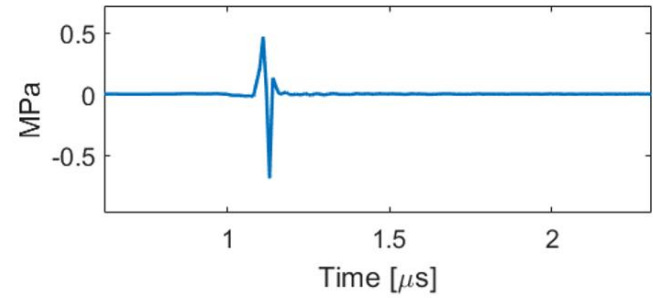

ii.

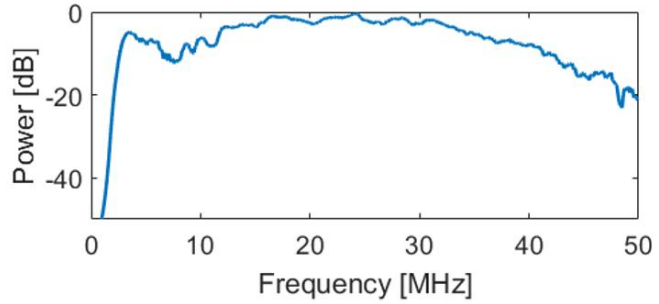

Fig. 2: (a) Schematic of the setup used for generation of optical ultrasound using in the fiber optic electrospun ultrasound transmitter, and pressure measurement using a calibrated needle hydrophone in a water bath, (b) Results of pressure measurement: i. ultrasound time trace, and ii. power spectrum of the measured optical ultrasound.

\section{Conclusion and future work}

To the best of our knowledge, we are the first to report electrospinning on an optical fiber endface with a scalable fabrication process and demonstrate its capability for optical ultrasound generation. Multiple ultrasound transmitters can be simultaneously fabricated using a single electrospinning and transfer step, followed by dip coating with PDMS elastomer. Ultrasound pressures within the range for clinical pulse-echo ultrasound were produced using the prepared fiber optic electrospun ultrasound transmitters.

The simple and controllable fabrication method for ultrasound transmitters used here could potentially be employed for making a wide variety of sensors. These transmitters will be used for optical ultrasound imaging by integrating with optical ultrasound detectors (for instance, Fabry Perot pressure sensors [12]) to form forward-looking all-optical ultrasound imaging probes. These ultrasound probes could assist with reduction in cost and usage complexity compared to conventional ultrasound transducers. The next steps include optimisation of the coating material and imaging of ex vivo tissue to ascertain its potential for medical imaging. The reported values of pressure and bandwidth are in the clinical range for pulse-echo ultrasound imaging. Other MIS procedures such as transseptal puncture, placenta imaging and plaque imaging in peripheral vessels could be explored using such fiber-optic ultrasound transmitters.

The paradigm of electrospinning and transfer of a functional polymer composite coating onto an optical fiber endface could also be extended to other fiber optic sensors. A CNT-PVA nanofibrous composite infiltrated with PDMS was used as the active element in the above detailed optical ultrasound transmitter, but other applications could be explored by modifying the electrospinning precursor in the fabrication method, and/or conjugating the nanofibers with active biomolecules or ligand groups. This could be useful in areas including but not limited to optical or chemical sensing and targeted in vivo drug delivery, with the use of appropriate alternate constituent composite materials, but the same fabrication methodology. 


\section{References}

1. G. Wild and S. Hinckley, "Acousto-ultrasonic optical fiber sensors: overview and state-of-the-art," IEEE Sensors Journal (2008).

2. H. Won Baac, J. G. J. Ok, H. J. H. Park, T. Ling, S. S. L. Chen, A. J. Hart, L. J. Guo, H. Baac, J. G. J. Ok, H. J. H. Park, T. Ling, S. S. L. Chen, H. Won Baac, J. G. J. Ok, H. J. H. Park, T. Ling, S. S. L. Chen, A. J. Hart, and L. J. Guo, "Carbon nanotube composite optoacoustic transmitters for strong and high frequency ultrasound generation," Applied Physics Letters 97, 38-41 (2010).

3. S. Noimark, R. J. Colchester, B. J. Blackburn, E. Z. Zhang, E. J. Alles, S. Ourselin, P. C. Beard, I. Papakonstantinou, I. P. Parkin, and A. E. Desjardins, "CarbonNanotubePDMS Composite Coatings on Optical Fibers for AllOptical Ultrasound Imaging,” Advanced Functional Materials 26, 83908396 (2016).

4. R. J. Colchester, E. Z. Zhang, C. A. Mosse, P. C. Beard, I. Papakonstantinou, and A. E. Desjardins, "Broadband miniature optical ultrasound probe for high resolution vascular tissue imaging,” Biomedical Optics Express 6, 1502-1511 (2015).

5. B.-Y. Hsieh, J. Kim, J. Zhu, S. Li, X. Zhang, and X. Jiang, "A laser ultrasound transducer using carbon nanofiberspolydimethylsiloxane composite thin film," Applied Physics Letters 106, 021,902 (2015).

6. H. W. Baac, J. G. Ok, T. Lee, and L. J. Guo, "Nano-structural characteristics of carbon nanotubepolymer composite films for high-amplitude optoacoustic generation," Nanoscale 7, 14,460-14,468 (2015).

7. Y. Murakami, E. Einarsson, T. Edamura, and S. Maruyama, "Polarization dependent optical absorption properties of singlewalled carbon nanotubes and methodology for the evaluation of their morphology," Carbon 43, 2664-2676 (2005).

8. C. Ni and P. R. Bandaru, "Enhanced optical absorption cross-section characteristics of multi-wall carbon nanotubes," Carbon 47, 2898-2903 (2009).

9. J. Vilčáková, R. Moučka, P. Svoboda, M. Ilčíková, N. Kazantseva, M. Hřibová, M. Mičušík, and M. Omastová, "Effect of Surfactants and manufacturing methods on the electrical and thermal conductivity of carbon nanotube/silicone composites," Molecules 17, 13,157-13,174 (2012).

10. R. Poduval, S. Noimark, and R. Colchester, “Optical fiber ultrasound transmitter with electrospun carbon nanotube-polymer composite," Applied Physics (2017).

11. R. J. Colchester, C. A. Mosse, D. S. Bhachu, J. C. Bear, C. J. Carmalt, I. P. Parkin, B. E. Treeby, I. Papakonstantinou, and A. E. Desjardins, "Laser-generated ultrasound with optical fibres using functionalised carbon nanotube composite coatings," Applied Physics Letters 104, 173,502 (2014).

12. E. Zhang, J. Laufer, and P. Beard, "Backward-mode multiwavelength photoacoustic scanner using a planar Fabry-Perot polymer film ultrasound sensor for high-resolution three-dimensional imaging of," Applied optics 46, 561-577 (2008). 